\title{
Analysis and Simulation for State of Traffic Data based on VANET
}

\author{
Zou Chunran1, Chen Zehan1, Ma Weihua1, Liu Yanlin1 \\ 1.College of Computer Science and Technology, Nanjing University of Aeronautics and \\ Astronautics, Nanjing, 210016 China
}

Keywords: Self-organized network; Routing; Junction; Traffic

\begin{abstract}
Because of fast moving of VANETs node and dynamic topology structure, the traditional wireless self-organized routing protocol is difficult to be directly applied in VANETs. Therefore, the routing protocol of hybrid traffic information perception is proposed. Through the collection of real-time traffic information, road weight is evaluated. The hybrid traffic information fuses density and network traffic of road node. According to the weight of each section, select the best routing, so as to establish the reliable and stable routing. In the junction, select function node through effective mechanism, and fuse traffic information through function node. The simulation results show that compared with the traditional location routing protocol, the proposed HTAR scheme has a greater improvement in the data transmission rate, network throughput performance.
\end{abstract}

\section{Introduction}

VANETs is different from MANETs (Mobility Ad hoc Networks), VANETs nodes move fast, and network structure dynamics is strong. These features make traditional routing algorithm difficult to be directly applied to VANETs. In addition, the application environment of VANETs is diverse, which causes the node communication is opportunistic. If in the dense region of the nodes, the connectivity between vehicles is better, with end-to-end connection, but the probability of end-to-end connection drops rapidly in sparsely populated areas. Even if the time exists, its duration is very short. In addition, the average duration of the vehicle communication window is 15 seconds [3], and makes two cars interaction time very short. Due to the special features of VANETs, in the design of VANETs routing protocol, systematic thinking is required, and limited network information is efficiently used, to improve the robustness and stability of routing.

\section{Assumption}

(1)There is wireless communication equipment in all the nodes in the network. Any two nodes can communicate with each other in their communication ranges.

(2) Each node has GPS service, and can get their location information;

(3) Before sending the data to the destination node, the source node has obtained the current position of the target node through the location service;

(4) All nodes can get the street information of their current position, including a) road ID, and the length of the road; B) ID of each junction and the position of the junction; C) the topology of the road.

\section{Basic concepts}

(1) Neighbor message

In the TARP, each node periodically broadcasts message "HELLO" to neighbor, so as to update the information. By the latest information, the node can select the best forwarding node for the data transfer. HELLO message format is as shown in table 1.

Table 1 Hello message format

\begin{tabular}{|l|l|l|l|}
\hline RoadIDLocation( $\mathrm{x}, \mathrm{y})$ & At junction (Yor N) & TTLJ or Channel load \\
\hline
\end{tabular}

Wherein, RoadID is the number of road of this node, and the Location (x, y) represents node 
location. The At junction $(\mathrm{Y} / \mathrm{N})$ represents whether the node is at the junction. If the node is at a certain junction, the last frame represents TTLJ, which represents the time when its node stays at the junction, otherwise, the last frame represents Channel load, which represents the road congestion time of nodes.

Once the node receives "hello" message from one node, the node will store the information in the neighbor information table. The format is as shown in table 2.

Table 2 Storage neighbor information format

\begin{tabular}{|c|c|c|c|c|c|}
\hline NodeID & RoadID & Location(x,y) & At junction & TTLJ or Channel load & Time stamp \\
\hline 2 & 0012 & $(123.56,452.56)$ & $\mathrm{Y}$ & $23 \mathrm{~s}$ & 9.0 \\
\hline 4 & 0003 & $(128.56,432.43)$ & $\mathrm{N}$ & 0.6 & 14.0 \\
\hline
\end{tabular}

(2) Routing processing procedure

As with GSR, A-STAR, TARP uses the shortest path algorithm to calculate the routing. In the process of routing, the junction is seen as vertices, road as edge, and each edge has its own weight. For the given two points, the TARP can find out the best routing path.

When the data begins to transmit, the source node needs to establish routing. According to the node's position, the establishment of the routing is divided into two categories. Firstly, the nodes are in the road. When node in the road receives a data package, it first checks the package destination, if the node itself is not the destination node of the package, and then the node will seek for the forwarding nodes suitable for next-hop based on the package destination. Once successfully finding the forwarding node, the task of the node is finished, otherwise, the node stores data packages until it can get the next-hop node. Secondly, the node is at the junction. When the node at the junction node receives the data package, it first checks whether it is the destination of the package. If the node is not the destination of the package, it checks whether the package arrives at the junction for the first time. If it is the first time, the node will recalculate the routing immediately for this package and seek the best next-hop forwarding nodes. If the suitable next-hop forwarding node can't be found, the node will calculate the routing for this package, and continues to seek the next-hop forwarding nodes.

(3) Junction leader node (LN) selection

At each junction, a unique LN must be chosen. LN is mainly responsible for periodically collecting real-time traffic information from nearby road, and its task can be divided into the following three aspects:

1) Collect real-time information of road traffic and network traffic from nearby road;

2) According to the collected information, set up the weight of the road;

3) The weight data of each road will be distributed to the nodes nearby the junction, including LG nearby the junction.

In the TARP, the node has four different states, including sleep, monitor, compete and spread. When the node is not at the junction, it enters the sleep state. Once the node enters the junction, its state turns into the monitor. The node in the monitor sets a countdown timer with ${ }^{T_{m}}$ seconds, and its purpose is to monitor the presence of $\mathrm{LN}$ at the junction. $\mathrm{LN}$ should periodically distribute Weight Information Update (WIU) to neighbor nodes. If the node receives WIU within $T_{m}$, the node is still in the state of the monitor and resets the current counter. If the node doesn't receive WIU within $T_{m}$, it shows no LN currently, the node enters the compete state.

The node in the compete state begins to compete LN position with other nodes. By this time, each node sets $T_{C}$ countdown timer, and $T_{c}$ is calculated by formula (1).

$$
T_{C}=\max \left(T_{c}^{\text {th }}, 1 /\lceil T T L J / \Delta t\rceil\right)+\operatorname{rand}()
$$

Wherein, $T_{c}^{\text {th }}$ is the upper limit of $T_{c}$. rand ( ) represents the function which produces random number, and is used to reduce different nodes and set the same $T_{c}$ probability. $\lceil\cdot\rceil$ represents round numbers down. Combined with TTLJ, $\Delta t$ is used to specify the countdown time of each 
node. From formula (1), the greater the TTLJ is, the smaller the corresponding $T_{c}$ is. After the $T_{C}$ timing is completed, the node immediately sends new LN messages to the neighbor, and enters the spread state at the same time. The node receiving the news, will terminate the countdown timer and also returns to the monitor state.

The nodes in the spread state are LN, they periodically collect road information and distribute information to neighbor nodes. Before LN leaves the junction, it must choose an inheritance node. The node with the maximum TTLJ , is selected as inheritance node. TTLJ can be calculated by formula (2) :

$$
\operatorname{TTLJ}(t)=\frac{D(t)}{V(t)}
$$

Wherein, $D(t)$ represents the distance of the node leaving the junction, and ${ }^{V(t)}$ represents the speed of the node.

The values of each node TTLJ are roughly estimated by formula (2). The greater the value is, the longer the time of the node staying in the junction is. When the node selects the inheritance node, and leaves the junction, it enters the sleep state. Inheritance node also enters the spread state accordingly.

(4) Task implementation of LN

Each LN has three tasks which must be completed: collection of traffic information, evaluation of road weights as well as the road weight information transfer.

In the TARP, traffic information implies two kinds of information. One is the density of nodes in roads, another is the network traffic congestion information, characterized by the road load.

In order to collect real-time traffic Information, LN transfers Traffic Information Collection (TIC) data package to neighboring LN. The format of the TIC is as shown in table 3.

Table 3 TIC format

\begin{tabular}{|c|c|c|c|c|c|}
\hline $\begin{array}{c}\text { Junction ID } \\
\text { (Source) }\end{array}$ & $\begin{array}{c}\text { Junction ID } \\
\text { (Destination) }\end{array}$ & $\begin{array}{c}\text { Time } \\
\text { stamp }\end{array}$ & $\begin{array}{c}\text { Segment } \\
\text { ID }\end{array}$ & $\begin{array}{c}\text { Number of } \\
\text { Nodes }\end{array}$ & $\begin{array}{c}\text { Channel } \\
\text { Load }\end{array}$ \\
\hline
\end{tabular}

Wherein, two columns JunctionID represent the source junction, destination junction respectively. Numbers of Nodes represents the number of nodes of the section of this road. Channel Load represents the load of the section of this road. Segment ID represents section number of the road. Timestamp represents the time of producing TIC package.

\section{Simulation and performance analysis}

In order to evaluate proposed VANET performance of routing, Network Simulator 2.31 is adopted as the simulation platform. Simulation scenario uses TraNSmodel. The specific parameters of simulation are as shown in table 4.

Table 4 Simulation parameters

\begin{tabular}{ll}
\hline Simulation area & $3000 \mathrm{~m}^{*} 2000 \mathrm{~m}$ \\
Node transmission range & $250 \mathrm{~m}$ \\
NS2 MAC layer & IEEE 802.11 \\
Packet size & 128 bytes \\
Speed limit of road (m/s) & 30 \\
Sending rate of CBR & 0.25 \\
Interval of "Hello" message broadcasting(s) & 2 \\
Number of Nodes & $200^{\sim} 400$ \\
Number of intersections & 40 \\
Simulation times $(\mathrm{s})$ & 200 \\
\hline
\end{tabular}

In order to better analyze the performance of the proposed protocol, this paper analyzes the performance respectively following the data of node number from the date package delivery ration 
and throughput. At the same time, it compares with two typical protocols of GSR, GyTAR.

\section{Concluding remarks}

This paper puts forward the routing algorithm based on traffic information. The algorithm sets a functional node $\mathrm{LN}$ at each junction. LN is responsible for collecting real-time traffic information, and calculates the weights of each section. At the same time LN delivers the information to the neighbor nodes and nearby junction. In order to meet the dynamic characteristics of topology structure of VANETs, LN fuses the node density, and load information of each section into traffic information. Therefore, node can choose the best forwarding nodes, making the routing path more robust and more stable. At the same time, $\mathrm{LN}$ is selected by setting countdown timer scheme, so as to reduce network overhead. The simulation results show that proposed protocol has superior performance in data deliver rate, throughput, compared with GSR and GyTAR .

\section{Acknowledgement}

The research is supported by College student innovation training program, project approval number: 201510287069.

\section{References}

[1] Lin Y, Yang J, Lv Z, et al. A Self-Assessment Stereo Capture Model Applicable to the Internet of Things[J]. Sensors, 2015, 15(8): 20925-20944.

[2] Yang J, He S, Lin Y, et al. Multimedia cloud transmission and storage system based on internet of things[J]. Multimedia Tools and Applications, 2015: 1-16.

[3] Lv Z, Yin T, Han Y, et al. WebVR-web virtual reality engine based on P2P network[J]. Journal of Networks, 2011, 6(7): 990-998.

[4] Jinyu $\mathrm{Hu}$ and Zhiwei Gao. Distinction immune genes of hepatitis-induced heptatocellular carcinoma[J]. Bioinformatics, 2012, 28(24): 3191-3194. 